\title{
Partial Virological Response after 2 Years of Entecavir Therapy Increases the Risk of Hepatocellular Carcinoma in Patients with Hepatitis B Virus-Associated Cirrhosis
}

\author{
Seung Kak Shin ${ }^{1}$, Hyung Joon Yim², Jeong Han Kim³, Chan Uk Lee ${ }^{4}$, Jong Eun Yeon ${ }^{4}$, Sang Jun Suh², Young Kul \\ Jung ${ }^{2}$, Yun Soo Kim¹, Ju Hyun Kim¹, and Oh Sang Kwon ${ }^{1}$ \\ 1Department of Internal Medicine, Gachon University Gil Medical Center, Gachon University College of Medicine, Incheon, \\ ${ }^{2}$ Department of Internal Medicine, Korea University Ansan Hospital, Ansan, ${ }^{3}$ Department of Internal Medicine, Konkuk University \\ School of Medicine, and ${ }^{4}$ Department of Internal Medicine, Korea University Guro Hospital, Seoul, Korea
}

\section{Article Info}

Received February 27, 2020

Revised May 28, 2020

Accepted June 8, 2020

Published online November 2, 2020

\section{Corresponding Author}

Oh Sang Kwon

ORCID https://orcid.org/0000-0001-9981-3914

E-mail kos@gilhospital.com

Seung Kak Shin and Hyung Joon Yim contributed equally to this work as first authors.
Background/Aims: The clinical significance of partial virological response (PVR) in patients undergoing antiviral therapy is not well known. This study investigated whether PVR after 2 years of entecavir (ETV) therapy is associated with hepatocellular carcinoma (HCC) development in cirrhotic patients.

Methods: A total of 472 naïve patients with hepatitis B virus (HBV)-associated cirrhosis who were treated with ETV for at least 2 years were retrospectively enrolled. Clinical characteristics, laboratory data, PVR, and noninvasive fibrosis markers (aspartate aminotransferase to platelet ratio and FIB-4 index) at 2 years after ETV commencement were analyzed for HCC risk.

Results: After excluding those who developed HCC within 2 years of ETV therapy, 359 patients (mean age, $51 \pm 10$ years; male $64.3 \%$ ) were examined. During a median follow-up of 82 months, 80 patients developed HCC. In the univariate analysis, older age (hazard ratio [HR], 1.056; $p<0.001)$, PVR (HR, 2.536; $p=0.002)$, higher aspartate aminotransferase $(H R, 1.018 ; p=0.005)$, lower albumin level (HR, 0.463; $p<0.001)$, lower platelet count $(H R, 0.993 ; p=0.01)$, and higher FIB-4 index (HR, 1.141; $p<0.001)$ at 2 years after ETV commencement were risk factors for HCC. In the multivariate analysis, older age (HR, 1.046; 95\% confidence interval [Cl], 1.022 to 1.072; $\mathrm{p}<0.001)$, PVR (HR, 2.358; 95\% Cl, 1.310 to 4.245; $p=0.004)$, and higher FIB-4 index (HR, 1.103; $95 \% \mathrm{Cl}, 1.035$ to $1.177 ; p=0.003$ ) were independent risk factors.

Conclusions: PVR and higher FIB-4 index after 2 years of ETV therapy were independent risk factors for HCC. Therefore, efforts to accomplish a complete virological response and reduce the FIB-4 index should be made. (Gut Liver 2021;15:430-439)

Key Words: Partial virological response; Entecavir; Hepatocellular carcinoma; Hepatitis B virus; Liver cirrhosis

\section{INTRODUCTION}

One of the risk factors for hepatocellular carcinoma (HCC) development in patients with hepatitis B virus (HBV) infection is a higher serum HBV DNA level. ${ }^{1} \mathrm{Se}-$ rum HBV DNA can be effectively suppressed by nucleos $(\mathrm{t})$ ide analogues (NAs). Therefore, in the era of NA therapy, higher basal serum HBV DNA level is no longer a risk factor for HCC development. However, there have been reports that insufficient suppression of serum HBV DNA after NA therapy may increase the risk of $\mathrm{HCC}^{2-4}$

Except in cases of primary nonresponse or virological breakthrough due to resistance, cases of detectable serum HBV DNA after NA therapy can be divided into two categories. The first category represents cases in which the level of HBV DNA continuously decreases but is still detectable; the viral load is low and there is no complete virological response (partial virological response, PVR). 
The other category includes cases in which the level of HBV DNA continuously decreases to undetectable level (complete virological response, CVR), but thereafter low level of intermittent or persistent viremia are observed. The former condition is usually due to a high viral load at baseline. ${ }^{5}$ The level of HBV DNA slowly decreases to undetectable level while the original medication is continued. ${ }^{5}$ In the second condition, patients show variable patterns of viremia. In such cases, the cause of viremia when patients have adhered to NA therapy and there is no resistance to NAs, remains unclear. There are no comparable studies on the outcomes of these two categories of cases with respect to HCC development and other liver-related events. However, the second category cannot be defined easily because the timing and patterns of serum HBV DNA reappearance are unpredictable and variable, respectively. In addition, it is not possible to predict the reappearance of serum HBV DNA unless serum HBV DNA is checked until the last minute. Therefore, the first category is easy to define and thus the risk of HCC in such cases can be examined. However, some reports have already shown that PVR with 1 year of NA therapy does not increase the risk of HCC. ${ }^{6,7}$

PVR is also called persistent viremia, which has traditionally been defined as detectable HBV DNA after 48 weeks of NA therapy by the American Association for the Study of Liver Diseases (AASLD) guidelines for treatment of chronic hepatitis $\mathrm{B}(\mathrm{CHB}){ }^{8}$ However, this definition was used in an era of NA therapy with lower antiviral potency. In the era of higher potent NAs such as entecavir (ETV), tenofovir disoproxil fumarate (TDF), and tenofovir alafenamide fumarate, persistent viremia is defined as failure to achieve an undetectable HBV DNA level after 96 weeks of NA therapy. ${ }^{8}$

The AASLD 2018 hepatitis B guidance suggests that persons with persistent viremia undergoing ETV or TDF monotherapy continue monotherapy. ${ }^{8}$ However, there is insufficient data on whether it is better to continue original NAs or to switch/add another NA in order to prevent liverrelated events including HCC development.

The present study aimed to investigate whether PVR after 2 years of ETV therapy is associated with HCC development in patients with liver cirrhosis (LC). In addition, other variables at 2 years after ETV commencement were also examined for their association with HCC development.

\section{MATERIALS AND METHODS}

\section{Patients}

The present study used data from patients with HBVassociated cirrhosis who underwent 2 years of ETV therapy. ${ }^{9}$ The observational time was extended in order to identify the risk factors for HCC development. A total of 472 naïve patients with $\mathrm{HBV}$-associated cirrhosis who were treated with ETV for at least 2 years, were retrospectively enrolled in four tertiary hospitals between March 2007 and December 2012. These patients did not have autoimmune hepatitis or viral coinfection such as hepatitis $\mathrm{C}$ virus, hepatitis $\mathrm{D}$ virus, or human immunodeficiency virus. All patients had a hepatitis B surface antigen more than 6 months, had a serum HBV DNA level of $\geq 4 \log _{10}$ copies/ $\mathrm{mL}$, and had an alanine aminotransferase or aspartate aminotransferase (AST) level of $>40 \mathrm{IU} / \mathrm{mL}$ according to the regulations imposed by Korean National Health Insurance. After enrollment, patients meeting any of the followings criteria were excluded: (1) diagnosis of HCC before entry or within 2 years after ETV commencement; (2) diagnosis of other cancer types at any time (before or after ETV commencement); (3) noncompliance with ETV therapy for more than 2 consecutive months within 2 years after ETV commencement; and (4) presence of missing data. Finally, 359 treatment-naïve patients with $\mathrm{HBV}$-associated cirrhosis were enrolled and the development of HCC was retrospectively examined until December 2018 (Fig. 1). The end points of follow-up were defined as the date of final visit, death, liver transplantation, or HCC diagnosis, whichever came first.

This study was approved by the Institutional Review Board of Gachon University Gil Medical Center and of each participating hospital (IRB numbers: GCIRB2013-42, GBIRB2020-048). This study was conducted in accordance with the Declaration of Helsinki. The informed consents from patients were waived due to retrospective collection of data.

\section{Diagnosis of LC and HCC}

LC was diagnosed by liver biopsy or clinical findings, such as an irregular liver surface or splenomegaly on radiologic images, varices on endoscopy, and thrombocytopenia $\left(<150 \times 10^{3} / \mu \mathrm{L}\right){ }^{10}$ All patients underwent regular screening for the detection of HCC according to the guideline issued by the Korean Liver Cancer Study Group. Serum $\alpha$-fetoprotein levels and abdominal ultrasonography were performed at 4- to 6-month intervals. If a nodular lesion was detected on ultrasonography or the $\alpha$-fetoprotein level was elevated, additional imaging studies including dynamic computed tomography or magnetic resonance imaging were performed. HCC was diagnosed according to the guidelines issued by the Korean Liver Cancer Study Group. ${ }^{11}$ 


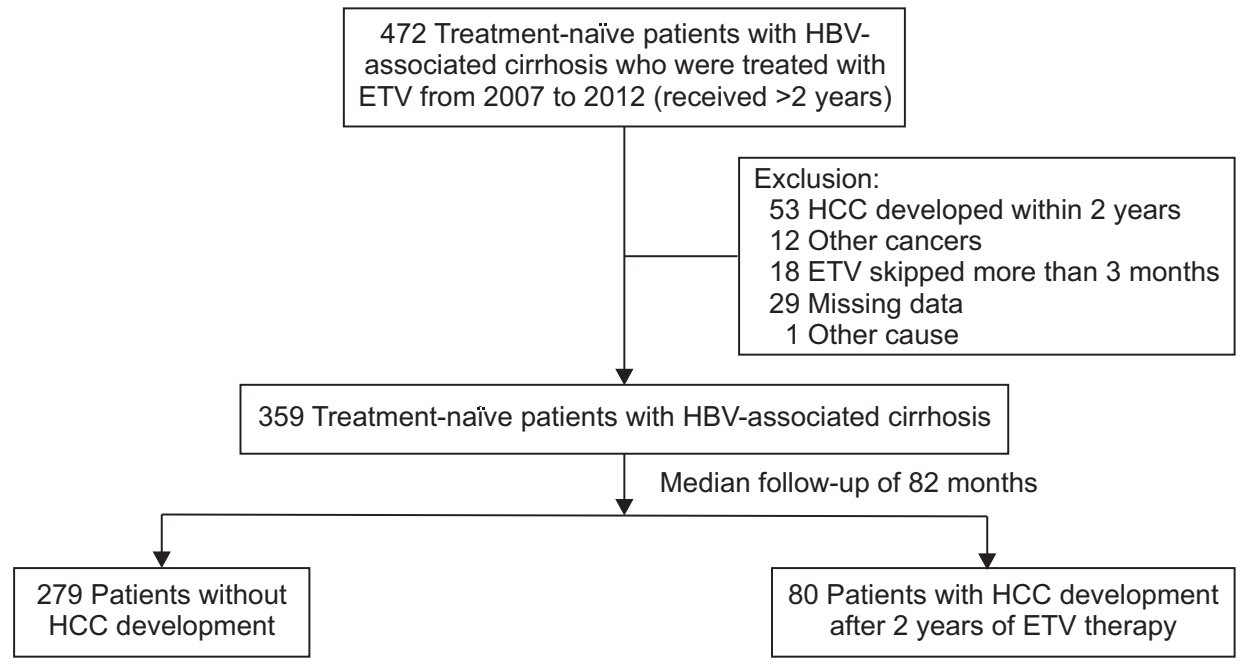

Fig. 1. Patient flowchart. HBV, hepatitis B virus; ETV, entecavir; HCC, hepatocellular carcinoma.

\section{Data collection at baseline and 2 years after ETV commencement}

Liver chemistries, platelet counts, international normalized ratio, hepatitis B envelop antigen (HBeAg), HBe antibody, and serum HBV DNA levels (COBAS TaqMan HBV Test v2.0, Roche Diagnostics, Branchburg, NJ, USA; low detection limit: 120 copies $/ \mathrm{mL}$ ) were collected. A serum HBV DNA level of $<120$ copies/mL was defined as the limit of detectability. PVR was defined as a detectable serum HBV DNA level $>120$ copies $/ \mathrm{mL}$ at 2 years after ETV commencement. The Model for End-Stage Liver Disease (MELD) score and proportion of Child-Pugh class were also collected both at baseline and 2 years after ETV commencement. The AST to platelet ratio index (APRI) and FIB-4 index were used to quantify the degree of liver fibrosis and were collected at both time points. These indices were calculated using the following formulas: APRI=[(AST/upper limit of normal)/platelet count $\left.\left(10^{9} / \mathrm{L}\right)\right]$ $\times 100$; FIB-4=age $($ years $) \times$ AST $(\mathrm{U} / \mathrm{L}) /\left[\right.$ platelet count $\left(10^{9} /\right.$ $\left.\mathrm{L}) \times(\text { alanine aminotransferase }[\mathrm{U} / \mathrm{L}])^{1 / 2}\right]$.

\section{Statistical analyses}

Values are presented as means \pm standard deviations or numbers of patients (\%). The Student t-test and paired ttest were used for continuous variables. The chi-square test was used for categorical variables. The cumulative incidences of HCC were computed using the KaplanMeier method. To identify risk factors of HCC, univariate and multivariate analyses were performed by the Cox regression analysis using variables at 2 years after ETV commencement. If any variables showed $p \leq 0.1$ in univariate analysis, they were used for multivariate analysis. The risks are expressed as hazard ratios (HRs) and 95\% confidence intervals (CIs). In addition, risk factors showing significance were stratified according to the values using the Kaplan-Meier method and compared using the logrank test. Statistical significance was accepted for $\mathrm{p}<0.05$. The analysis was performed using SPSS version 23.0 (IBM Corp., Armonk, NY, USA).

\section{RESULTS}

\section{Patient characteristics at baseline and 2 years after ETV commencement}

The characteristics at baseline and 2 years after ETV commencement are summarized in Table 1. The mean age was $51 \pm 10$ years, and 231 patients $(64.3 \%)$ were male. At 2 years after ETV commencement, liver chemistries, international normalized ratio, platelet count, proportion of Child-Pugh class, MELD score, APRI, and FIB-4 index all showed improvement compared to baseline values. Six patients (1.7\%) developed resistance to ETV during the study period. Switching of ETV to TDF or adding of TDF on ETV was done in those patients. Five patients received liver transplantation due to poor liver function and 28 patients died due to liver-related causes during the study periods.

\section{Comparison of patients' characteristics between CVR and PVR}

HBV DNAs were detectable in 35 patients (9.7\%) at 2 years after ETV commencement (PVR). The mean level of HBV DNA in patients with PVR was $2.7 \pm 1.0$ (range, 2.1 to 7.5) $\log _{10}$ copies $/ \mathrm{mL}$. They were continuously treated with ETV, if there was no resistance. Patients with PVR had higher male proportion, baseline HBV DNA, and positivity of HBeAg compared to patients with CVR (Table 2). However, there were no differences in age, liver chemistries, platelet counts, proportion of Child-Pugh class, MELD 
Table 1. Characteristics at Baseline and 2 Years after ETV Commencement

\begin{tabular}{|c|c|c|c|}
\hline Characteristics & Baseline ( $n=359)$ & At 2 year after ETV commencement ( $n=359$ ) & $\mathrm{p}$-value \\
\hline Age, yr & $51 \pm 10$ & & \\
\hline Male sex & 231 (64.3) & & \\
\hline AST, IU/L & $126 \pm 161$ & $34 \pm 14$ & $<0.001$ \\
\hline$A L T, I U / L$ & $130 \pm 171$ & $31 \pm 17$ & $<0.001$ \\
\hline Albumin, g/dL & $3.7 \pm 0.6$ & $4.1 \pm 0.5$ & $<0.001$ \\
\hline Total bilirubin, $\mathrm{mg} / \mathrm{dL}$ & $1.9 \pm 2.6$ & $1.3 \pm 1.0$ & $<0.001$ \\
\hline INR & $1.27 \pm 0.28$ & $1.14 \pm 0.57$ & $<0.001$ \\
\hline Platelet count, $\times 10^{3} / \mu \mathrm{L}$ & $102 \pm 41$ & $107 \pm 44$ & $<0.001$ \\
\hline HBeAg positivity & $177(49.3)$ & $127(37.7)$ & $<0.001$ \\
\hline HBV DNA, $\log _{10}$ copies $/ \mathrm{mL}$ & $7.0 \pm 1.2$ & $0.2 \pm 0.8$ & $<0.001$ \\
\hline CP class & & & $<0.001$ \\
\hline A & 254 (70.8) & $322(89.7)$ & \\
\hline B & 89 (24.8) & 34 (9.5) & \\
\hline C & $16(4.4)$ & $3(0.8)$ & \\
\hline MELD & $8.4 \pm 4.6$ & $6.2 \pm 4.2$ & $<0.001$ \\
\hline APRI & $3.6 \pm 4.6$ & $1.5 \pm 1.5$ & $<0.001$ \\
\hline FIB-4 index & $6.8 \pm 5.9$ & $3.9 \pm 2.7$ & $<0.001$ \\
\hline
\end{tabular}

Data are presented as mean \pm SD or number (\%).

ETV, entecavir; AST, aspartate aminotransferase; ALT, alanine aminotransferase; INR, international normalized ratio; HBeAg, hepatitis B e antigen; HBV, hepatitis B virus; CP, Child-Pugh; MELD, Model for End-Stage Liver Disease; APRI, aspartate transaminase to platelet ratio index.

Table 2. Comparison of Patients' Characteristics between CVR and PVR at 2 Years after ETV Commencement

\begin{tabular}{|c|c|c|c|}
\hline Characteristics & CVR $(n=324)$ & PVR (n=35) & $\mathrm{p}$-value \\
\hline Age, yr & $53 \pm 9$ & $54 \pm 11$ & 0.424 \\
\hline Male sex & 203 (62.7) & $28(80.0)$ & 0.042 \\
\hline AST, IU/L & $33 \pm 13$ & $40 \pm 21$ & 0.051 \\
\hline ALT, IU/L & $30 \pm 16$ & $35 \pm 20$ & 0.161 \\
\hline Total bilirubin, mg/dL & $1.3 \pm 1.0$ & $1.3 \pm 0.7$ & 0.687 \\
\hline Albumin, g/dL & $4.1 \pm 0.5$ & $3.9 \pm 0.6$ & 0.024 \\
\hline INR & $1.1 \pm 0.6$ & $1.1 \pm 0.2$ & 0.904 \\
\hline Platelet count, $\times 10^{3} / \mu \mathrm{L}$ & $107 \pm 45$ & $104 \pm 40$ & 0.731 \\
\hline HBV DNA, $\log _{10}$ copies $/ \mathrm{mL}$ & Undetectable & $2.7 \pm 1.0$ & \\
\hline Basal HBV DNA & $6.9 \pm 1.2$ & $7.6 \pm 1.2$ & 0.001 \\
\hline HBeAg positivity & 107/305 (35.1) & $20 / 34(58.9)$ & 0.002 \\
\hline CP class & & & 0.072 \\
\hline A & 294 (90.7) & $28(80.0)$ & \\
\hline B & 27 (8.3) & $7(20.0)$ & \\
\hline C & $3(1.0)$ & 0 & \\
\hline MELD score & $6.3 \pm 4.3$ & $5.6 \pm 3.5$ & 0.412 \\
\hline APRI & $1.5 \pm 1.5$ & $1.6 \pm 1.1$ & 0.563 \\
\hline FIB-4 index & $3.8 \pm 2.7$ & $4.5 \pm 3.1$ & 0.129 \\
\hline
\end{tabular}

Data are presented as mean \pm SD or number $(\%)$.

CVR, complete virological response; PVR, partial virological response; ETV, entecavir; AST, aspartate aminotransferase; ALT, alanine aminotransferase; INR, international normalized ratio; HBV, hepatitis B virus; HBeAg, hepatitis B e antigen; CP, Child-Pugh; MELD, Model for End-Stage Liver Disease; APRI, aspartate transaminase to platelet ratio index.

score, APRI, and FIB-4 index between the two groups. In 25 patients with PVR, HBV DNA levels (baseline: 7.7 [5.1 to 9.0$] \log _{10}$ copies/mL and at PVR: 2.4 [2.1 to 4.7$] \log _{10}$ copies $/ \mathrm{mL}$ ) continuously decreased until they reached undetectable levels during 0.5 to 3 years. Seven patients with PVR showed persistently detectable HBV DNA at low levels (baseline HBV DNA: 5.0 [2.5 to 5] $\log _{10}$ copies $/ \mathrm{mL}$ and HBV DNA at PVR: 2.4 [2.2 to 2.9$] \log _{10}$ copies/mL) during
3.2 to 10.7 years of follow-up. The levels of HBV DNA in three patients with PVR (baseline HBV DNA: 8.0 [4.3 to 10.1] $\log _{10}$ copies/mL and HBV DNA at PVR: 2.8 [2.7 to 7.5] $\log _{10}$ copies $/ \mathrm{mL}$ ) showed virological breakthrough with resistance at 2, 3, 4 years after ETV commencement, respectively. 


\section{Cumulative incidence of HCC}

Of the 359 patients with HBV-associated cirrhosis, 80 (22.3\%) developed HCC after 2 years of ETV therapy during median 82 months (range, 24 to 133 months) of followup periods. The cumulative incidence of HCC is shown in Fig. 2. According to the modified union of international cancer control classification, 94\% of HCC patients had stage I or II diseases.

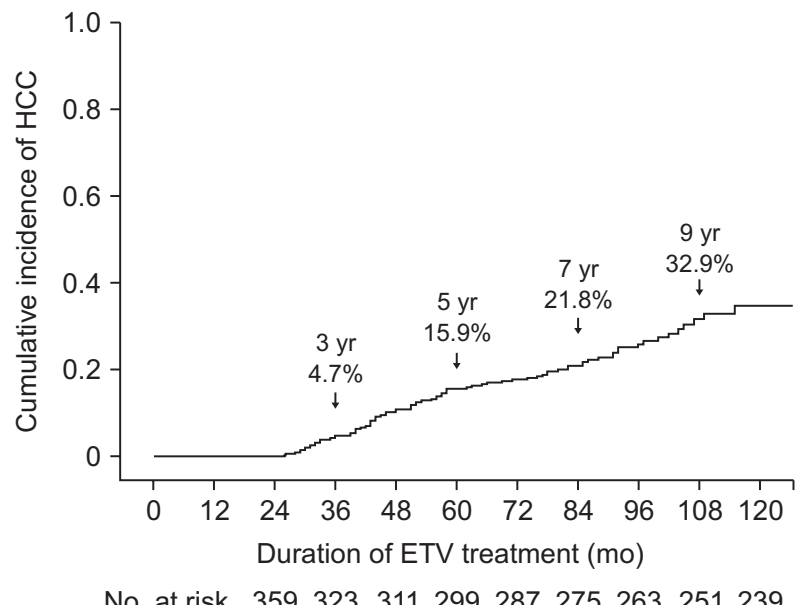

Fig. 2. Cumulative incidence of hepatocellular carcinoma (HCC) in 359 patients with hepatitis $B$ virus-associated cirrhosis.

ETV, entecavir.

\section{Comparison of characteristics at 2 years after ETV commencement between cirrhotic patients without HCC (LC group) and those with HCC (HCC group)}

The HCC group ( $\mathrm{n}=80)$ had older age, higher proportion of male, higher AST level, lower albumin level, lower platelet count, higher frequency of PVR, and higher FIB-4 index than those in the LC group $(n=279)$ (Table 3). However, the HBeAg positivity, proportion of Child-Pugh class, MELD score, and APRI were not different between the two groups.

\section{Risk factors of HCC by Cox regression analysis based on the data at 2 years after ETV commencement}

Univariate analysis identified the following as significant risk factors of HCC: older age, higher AST level, lower albumin level, lower platelet count, presence of PVR, and higher FIB-4 index. However, gender, HBeAg positivity, Child-Pugh class, MELD score, and APRI were not associated with HCC development. Multivariate analysis revealed the following as significant risk factors of HCC: older age (HR, 1.046; 95\% CI, 1.022 to 1.072; $\mathrm{p}<0.001$ ), presence of PVR (HR, 2.358; 95\% CI, 1.310 to 4.245; $\mathrm{p}=0.004$ ), and higher FIB-4 index (HR, 1.103; 95\% CI, 1.035 to 1.177; $\mathrm{p}=0.003$ ) (Table 4).

In patients with PVR, the incidence of HCC development in patients who finally reached undetectable DNA levels, patients with persistent low-level viremia, and patients who showed viral breakthrough were $40.0 \%(10 / 25)$,

Table 3. Comparison of Characteristics at 2 Years after ETV Commencement between LC Patients with and without HCC Development

\begin{tabular}{|c|c|c|c|}
\hline Characteristics & Non-HCC (n=279) & $\mathrm{HCC}(\mathrm{n}=80)$ & $\mathrm{p}$-value \\
\hline Age, yr & $52 \pm 9$ & $57 \pm 8$ & $<0.001$ \\
\hline Male sex & $176(63.1)$ & 55 (68.8) & 0.031 \\
\hline AST, IU/L & $33 \pm 14$ & $40 \pm 13$ & 0.019 \\
\hline ALT, IU/L & $31 \pm 18$ & $31 \pm 13$ & 0.929 \\
\hline Albumin, g/dL & $4.1 \pm 0.5$ & $4.0 \pm 0.5$ & 0.011 \\
\hline Total bilirubin, $\mathrm{mg} / \mathrm{dL}$ & $1.2 \pm 0.7$ & $1.4 \pm 1.5$ & 0.333 \\
\hline INR & $1.14 \pm 0.64$ & $1.14 \pm 0.22$ & 0.994 \\
\hline Platelet count, $\times 10^{3} / \mu \mathrm{L}$ & $110 \pm 44$ & $96 \pm 42$ & 0.018 \\
\hline HBeAg positivity & 98 (37.0) & 29 (40.3) & 0.681 \\
\hline Partial virological response & $21(7.5)$ & 14 (17.5) & 0.023 \\
\hline CP class & & & 0.882 \\
\hline A & 251 (90.0) & 71 (88.8) & \\
\hline B & $26(9.3)$ & $8(10.0)$ & \\
\hline C & $2(0.7)$ & $1(1.2)$ & \\
\hline MELD & $6.3 \pm 4.5$ & $6.0 \pm 3.2$ & 0.612 \\
\hline APRI & $1.5 \pm 1.6$ & $1.5 \pm 1.1$ & 0.954 \\
\hline FIB-4 index & $3.5 \pm 2.3$ & $5.1 \pm 3.6$ & $<0.001$ \\
\hline
\end{tabular}

Data are presented as mean \pm SD or number (\%).

ETV, entecavir; LC, liver cirrhosis; HCC, hepatocellular carcinoma; AST, aspartate aminotransferase; ALT, alanine aminotransferase; INR, international normalized ratio; HBeAg, hepatitis B e antigen; CP, Child-Pugh; MELD, Model for End-Stage Liver Disease; APRI, aspartate transaminase to platelet ratio index. 
Table 4. Univariate and Multivariate Analysis for the Risk Factors of HCC Based on Variables at 2 Years after ETV Commencement

\begin{tabular}{|c|c|c|c|c|}
\hline \multirow{2}{*}{ Variable } & \multicolumn{2}{|c|}{ Univariate analysis } & \multicolumn{2}{|c|}{ Multivariate analysis } \\
\hline & $\mathrm{HR}(95 \% \mathrm{Cl})$ & $\mathrm{p}$-value & $\operatorname{HR}(95 \% \mathrm{Cl})$ & p-value \\
\hline Age, yr & 1.056 (1.031-1.082) & $<0.001$ & 1.046 (1.022-1.072) & $<0.001$ \\
\hline Male sex & 1.325 (0.825-2.127) & 0.197 & & \\
\hline AST, IU/L & 1.018 (1.005-1.030) & 0.005 & 1.006 (0.989-1.023) & 0.507 \\
\hline ALT, IU/L & 1.001 (0.988-1.014) & 0.899 & & \\
\hline Albumin, g/dL & $0.463(0.301-0.713)$ & $<0.001$ & $1.016(0.556-1.856)$ & 0.978 \\
\hline Total bilirubin, mg/dL & 1.109 (0.940-1.309) & 0.220 & & \\
\hline Platelet counts, $\times 10^{3} / \mu \mathrm{L}$ & 0.993 (0.987-0.998) & 0.010 & 0.997 (0.990-1.005) & 0.535 \\
\hline Partial virological response & $2.536(1.423-4.520)$ & 0.002 & $2.358(1.310-4.245)$ & 0.004 \\
\hline \multicolumn{5}{|l|}{ CP class } \\
\hline A & Reference & & & \\
\hline B & $1.422(0.683-2.960)$ & 0.347 & & \\
\hline C & 1.684 (0.233-12.145) & 0.605 & & \\
\hline MELD & $0.992(0.938-1.050)$ & 0.791 & & \\
\hline APRI & 1.160 (0.990-1.358) & 0.066 & 0.958 (0.751-1.223) & 0.732 \\
\hline FIB-4 index & $1.141(1.080-1.206)$ & $<0.001$ & 1.103 (1.035-1.177) & 0.003 \\
\hline FIB-4 $\geq 3.25$ & 2.868 (1.804-4.558) & $<0.001$ & $2.243(1.391-3.616)$ & 0.001 \\
\hline
\end{tabular}

HCC, hepatocellular carcinoma; ETV, entecavir; HR, hazard ratio; Cl, confidence interval; AST, aspartate aminotransferase; ALT, alanine aminotransferase; CP, Child-Pugh; MELD, Model for End-Stage Liver Disease; APRI, aspartate transaminase to platelet ratio index.

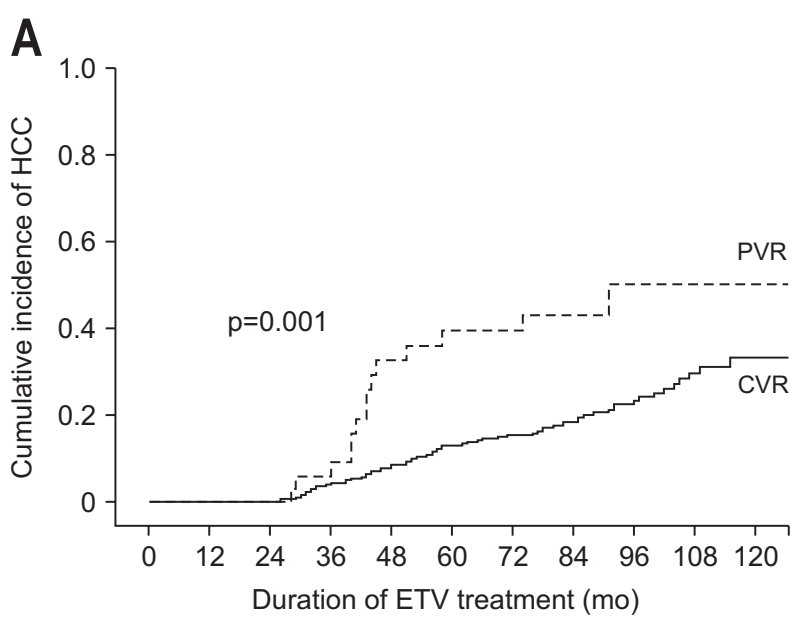

No. at risk

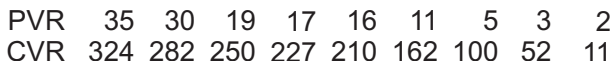

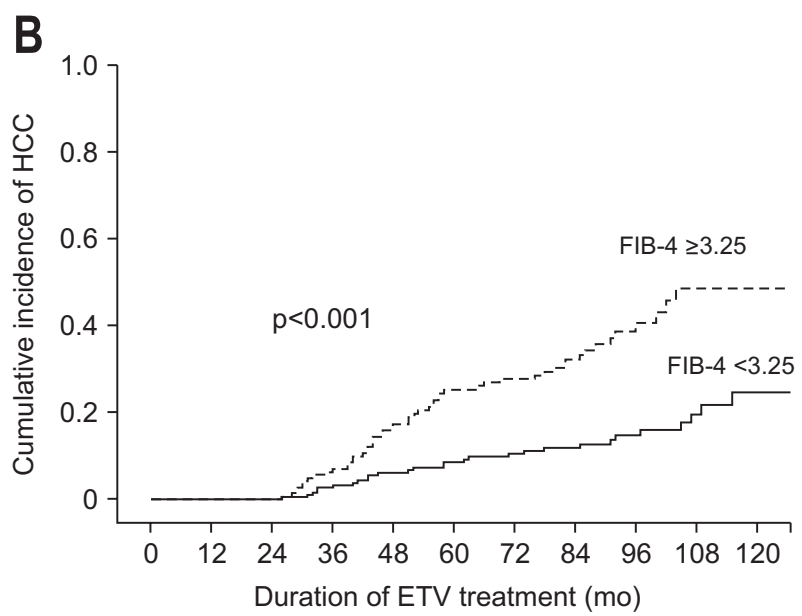

No. at risk

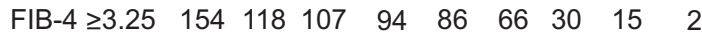
FIB-4 <3.25 $200177 \quad 154 \quad 144 \quad 135 \quad 103 \quad 74 \quad 38 \quad 11$

Fig. 3. Cumulative incidence of hepatocellular carcinoma (HCC) according to PVR (A) or FIB-4 $\geq 3.25$ (B).

ETV, entecavir; PVR, partial virological response; CVR, complete virological response.

$42.9 \%(3 / 7)$, and $33.3 \%(1 / 3)$, respectively. There was no statistical difference in HCC development among the three groups.

\section{Cumulative incidence of HCC according to PVR or FIB-4 index (cutoff=3.25)}

Fig. 3A shows the cumulative incidence of HCC according to the presence of PVR after 2 years of ETV therapy. The cumulative incidence of HCC in patients with PVR was $9.0 \%$ at 3 years, $39.5 \%$ at 5 years, $43.1 \%$ at 7 years, and $50.2 \%$ at 9 years. The cumulative incidence of HCC in pa- tients with CVR after 2 years of ETV therapy was $4.3 \%$ at 3 years, $13.0 \%$ at 5 years, $18.5 \%$ at 7 years, and $29.7 \%$ at 9 years. The cumulative incidence of HCC was higher in patients with PVR than in those with CVR ( $p=0.001)$. Fig. 3B shows the cumulative incidence of HCC according to FIB4 index (cutoff $=3.25$ ) after 2 years of ETV therapy. The cumulative incidence of HCC in patients with FIB-4 index $\geq 3.25$ was $7 \%$ at 3 years, $25.2 \%$ at 5 years, $32.2 \%$ at 7 years, and $48.6 \%$ at 9 years. The cumulative incidence of HCC in patients with FIB-4 index $<3.25$ was $3.2 \%$ at 3 years, $8.6 \%$ at 5 years, $11.9 \%$ at 7 years, and $19.6 \%$ at 9 years. The 
cumulative incidences of HCC was higher in patients with FIB-4 index $\geq 3.25$ than in those with FIB-4 index $<3.25$ $(\mathrm{p}<0.001)$.

\section{Cumulative incidence of $\mathrm{HCC}$ according to the combination of the two risk factors (PVR and FIB-4 index)}

Fig. 4 shows the cumulative incidence of HCC according to the following: group A, CVR and FIB-4 index $<3.25$; group $\mathrm{B}, \mathrm{CVR}$ and FIB- 4 index $\geq 3.25$; group C, PVR and FIB-4 index <3.25; and group D, PVR and FIB-4 index $\geq 3.25$. The cumulative incidence of HCC in group A was $2.9 \%$ at 3 years, $6.2 \%$ at 5 years, $9.1 \%$ at 7 years, and $16.4 \%$ at 9 years. The cumulative incidence of HCC in group A (CVR and FIB-4 index <3.25) was lower than that in groups $\mathrm{B}, \mathrm{C}$, and $\mathrm{D}($ all $\mathrm{p}<0.001)$.

\section{DISCUSSION}

We previously showed that ETV therapy can improve liver function, suppress HBV replication, and decrease the noninvasive fibrosis index after 2-year therapy. ${ }^{9}$ At 2 years after ETV commencement, most of the patients showed CVR except 35 patients.

In the present study, PVR was defined as a detectable HBV DNA level at 2 years after ETV commencement. Persistent viremia, PVR, and suboptimal response are all synonymous terms used by AASLD, European Association for the Study of the Liver, and Asian Pacific Association for the Study of Liver, respectively. They are terms used to indicate incomplete virological response, which is defined

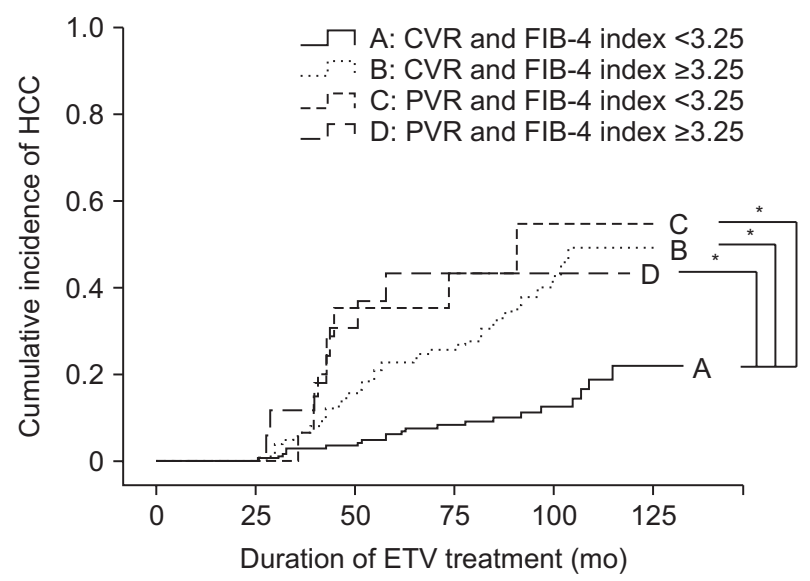

Fig. 4. Cumulative incidence of hepatocellular carcinoma (HCC) according to the combination of the two risk factors (PVR and FIB-4 index 23.25 ).

ETV, entecavir; PVR, partial virological response; CVR, complete virological response. ${ }^{*} \mathrm{p}<0.001$. as a decrease in HBV DNA level of more than $1 \log _{10} \mathrm{IU} /$ $\mathrm{mL}$ but a detectable HBV DNA at a certain time point after NA therapy. ${ }^{5,8,12}$ The definition of this time point differs among the different guidelines: it is 96 weeks in AASLD, 12 months in the European Association for the Study of the Liver, and 6 months in Asian Pacific Association for the Study of Liver guidelines. ${ }^{5,8,12}$ Thus, although the eventual meaning of an incomplete virological response is nearly the same among the different guidelines, the time point of reference used for its definition varies. However, in the current era of highly effective NAs, the AASLD guideline seems to be the most reasonable one for defining incomplete virological response. ${ }^{5,8,12}$

The clinical significance of a PVR with respect to the outcome of CHB is not clear. Therefore, there is no consensus on whether it is advisable to continue the original NAs or to switch/add other NAs when a PVR is observed. Several guideline committees have provided different recommendations. The European Association for the Study of the Liver guideline provided a detailed guideline indicating that the HBV DNA levels at 12 months and declining pattern of HBV DNA after therapy must be considered simultaneously. If HBV DNA levels continue to decline, original NAs may be used continuously. However, if HBV DNA levels show plateau, NAs could be switched or other NAs could be added. ${ }^{5}$ In the present study, most patients with a PVR showed a slow but continuous reduction in the levels of serum HBV DNA over time without changing or adding NAs. Therefore, considering only the virological response, continuing the original NAs appears reasonable. However, when the risk for HCC development is considered, continuing the original NAs may not be reasonable owing to the higher incidence of HCC in patients with a PVR than in those with CVR.

Several reports show that the suppression of HBV replication with NAs reduces the risk of HCC. ${ }^{11,13,14}$ However, some patients showed PVR or intermittent viremia on NA therapy. The levels of viremia in such cases were usually low $(<2,000 \mathrm{IU} / \mathrm{mL})$, if they were not caused by a primary nonresponse or presence of resistance. The effect of such low viremia on HCC development is still under debate. We have previously reported that PVR with 1 year of ETV therapy did not significantly increase the risk of HCC when compared to CVR. ${ }^{7}$ Wong et al. ${ }^{6}$ also reported that PVR with 1 year of NA (several kinds of NAs) therapy did not significantly increase the risk of HCC, although they did not carry out a detailed analysis of HCC development. However, the present study shows that PVR after 2 years of ETV therapy increases the risk of HCC. Based on these results, it appears that the time point of PVR influences the results of studies on HCC development. 
Kim et al. ${ }^{2}$ reported that the PVR or intermittent low level of viremia after ETV therapy increases the risk of HCC in patients with LC when compared to CVR. However, they did not define the time point of PVR and did not distinguish between persistent and intermittent viremia. In fact, the nature of persistent viremia may be different from that of intermittent viremia. Persistent viremia may be due to innate viral and host factors, whereas most cases of intermittent viremia may be related to therapy compliance. In addition, it is not possible to predict whether an individual has persistent or intermittent viremia until viremia is examined continuously. However, the present study used a clear time point to define PVR and thus assessed the risk of HCC after 2 years of ETV therapy.

It is unclear why PVR increases the risk of HCC. In the present study, the pattern of HCC development demonstrated that patients with PVR show a sharp increase in the incidence of HCC within 2 to 3 years after the 2 years of ETV therapy, and this is followed by a slow increase in the incidence over time. In contrast, patients with CVR showed a slow increase in the incidence of HCC. The different patterns of HCC development suggest that incomplete inhibition of HBV DNA replication may significantly influence HCC development until serum HBV DNA is cleared. As a matter of fact, most patients with PVR were cleared of the serum HBV DNA during 0.5 to 3 years. Therefore, to decrease the risk of HCC as early as possible, HBV DNA should be completely suppressed.

In the present study, the FIB-4 index was found to be a strong risk factor for HCC development in the univariate and multivariate analyses. An FIB- 4 index $\geq 3.25$ at 2 years after ETV commencement was significantly associated with a higher risk of HCC. Although all patients had LC, higher MELD or higher Child-Pugh class were not risk factors for HCC development. These results suggest that FIB-4 index is an independent risk factor for HCC development in patients with HBV-associated LC on ETV therapy.

A high FIB-4 index was a predictive factor for HCC development not only in patients not undergoing NA therapy ${ }^{15-17}$ but also in those undergoing NA therapy. ${ }^{18,19}$ Most FIB-4 studies in patients with $\mathrm{CHB}$ have been performed in a pretreatment rather than in a posttreatment state. ${ }^{20}$ However, Tada et al. ${ }^{19}$ reported that a higher FIB- 4 index at 24 weeks after NA commencement is a risk factor for HCC development. Similarly, the present study also demonstrated that higher FIB- 4 index at 2 years after ETV commencement is associated with HCC development. Because FIB-4 index includes liver transaminases that are involved in liver inflammation, the initial FIB-4 index may not reflect the exact fibrosis stage due to the presence of active inflammation at enrollment. In the present study, higher baseline FIB-4 index was not associated with the risk of HCC (data not shown). Therefore, the FIB-4 index after transaminase stabilization by NAs may be more suitable to evaluate fibrosis stage and the risk of HCC. Although we did not analyze FIB-4 indices at other times after ETV commencement for the risk of HCC, higher FIB-4 index is likely a consistent risk factor of HCC, independent of the time point of measurement. Because the FIB- 4 index is a noninvasive marker of fibrosis, the higher FIB-4 index indicates a more advanced stage of liver fibrosis, which itself is a strong risk factor of HCC. In addition, the formula of FIB-4 index includes age, which is a major risk factor of HCC. ${ }^{18}$ Taken together, this indicates that the FIB-4 index may be the strongest risk factor of HCC rather than a simple fibrosis marker.

APRI has also been shown to be a valuable fibrosis marker in patients with $\mathrm{CHB}^{20,21}$ However, it remains unclear whether it is a useful predictor for HCC development in patients with CHB. ${ }^{16,18}$ APRI might be less powerful for predicting the risk of HCC than the FIB-4 index, because its formula does not include age. In the present study, APRI had borderline significance for predicting the risk of HCC in the univariate analysis and was not a significant risk factor in the multivariate analysis.

The present study had several limitations. First, it was a retrospective study based on previously published data. Therefore, several factors influencing the risk of HCC and medication compliance after 2 years of ETV therapy were not examined. Second, the majority of cirrhotic cases were diagnosed by clinical findings. Although the FIB-4 index and APRI are not perfect diagnostic indicators of LC, most patients had an FIB-4 index $>3.25$ or APRI $>2.0$, which provide good sensitivity and specificity for diagnosing advanced fibrosis or cirrhosis. Third, the detectability of serum HBV DNA throughout the study period (intermittent viremia) in patients with CVR was not analyzed. Further studies are needed to compare the effect of PVR and intermittent viremia after CVR on HCC development during ETV therapy. Forth, the present study did not switch to or add other NAs in cases of PVR after 2 years of ETV therapy. Several studies already demonstrated that TDFbased alternative therapies were effective in patients with PVR. ${ }^{22,23}$ However, a prospective study is needed to investigate whether the incidence of HCC decreases when treatment is switched to other NAs or other NAs are added in cases of PVR. Fifth, the present study used only ETV as a first choice for treatment of naïve patients. Other NAs may have different result. Choi et al. ${ }^{24}$ reported that there was difference in the HCC risk between patients using TDF and ETV in spite of similar efficacy. Therefore, the result of 
present study applies only to the patients using ETV.

In conclusion, PVR and higher FIB- 4 index after 2 years of ETV therapy were significant independent risk factors of HCC. If there is a CVR and the FIB-4 index is less than 3.25 , the risk of HCC is lowest. Although there were several studies that each PVR and high FIB-4 index were associated with HCC risk, ${ }^{2,19,25}$ the present study simultaneously analyzed the two factors as a risk of HCC. Therefore, PVR and FIB-4 index must be taken account for the estimation of HCC risk in cases of antiviral therapy.

\section{CONFLICTS OF INTEREST}

No potential conflict of interest relevant to this article was reported.

\section{AUTHOR CONTRIBUTIONS}

Design this study: O.S.K., H.J.Y. Data collection and analysis: S.K.S., J.H.K., C.U.L., S.J.S., Y.K.J. Writing - original draft: O.S.K., S.K.S., H.J.Y. Writing - review and editing: J.E.Y., Y.S.K., J.H.K. Approval of final manuscript: all authors.

\section{ORCID}

Seung Kak Shin https://orcid.org/0000-0002-5120-5300 Hyung Joon Yim https://orcid.org/0000-0002-6036-2754 Jeong Han Kim https://orcid.org/0000-0002-8383-8524 Chan Uk Lee https://orcid.org/0000-0003-2053-3894 Jong Eun Yeon https://orcid.org/0000-0002-0510-7371 Sang Jun Suh https://orcid.org/0000-0003-4128-3732 Young Kul Jung https://orcid.org/0000-0002-6566-1382 Yun Soo Kim https://orcid.org/0000-0003-2380-1217 Ju Hyun Kim https://orcid.org/0000-0001-8981-135X Oh Sang Kwon https://orcid.org/0000-0001-9981-3914

\section{REFERENCES}

1. Chen CJ, Yang HI, Su J, et al. Risk of hepatocellular carcinoma across a biological gradient of serum hepatitis $\mathrm{B}$ virus DNA level. JAMA 2006;295:65-73.

2. Kim JH, Sinn DH, Kang W, et al. Low-level viremia and the increased risk of hepatocellular carcinoma in patients receiving entecavir treatment. Hepatology 2017;66:335-343.

3. Kim SS, Hwang JC, Lim SG, Ahn SJ, Cheong JY, Cho SW. Effect of virological response to entecavir on the development of hepatocellular carcinoma in hepatitis B viral cirrhotic patients: comparison between compensated and decompensated cirrhosis. Am J Gastroenterol 2014;109:1223-1233.

4. Terrault $\mathrm{N}$. Is low level viremia acceptable during antiviral therapy of patients with $\mathrm{HBV}$ infection and decompensated cirrhosis? Clin Gastroenterol Hepatol 2018;16:1876-1878.

5. European Association for the Study of the Liver. EASL 2017 clinical practice guidelines on the management of hepatitis B virus infection. J Hepatol 2017;67:370-398.

6. Wong GL, Chan HL, Tse YK, et al. Normal on-treatment ALT during antiviral treatment is associated with a lower risk of hepatic events in patients with chronic hepatitis B. J Hepatol 2018;69:793-802.

7. Kim EJ, Yeon JE, Kwon OS, et al. Rapid alanine aminotransferase normalization with entecavir and hepatocellular carcinoma in hepatitis B virus-associated cirrhosis. Dig Dis Sci 2017;62:808-816.

8. Terrault NA, Lok ASF, McMahon BJ, et al. Update on prevention, diagnosis, and treatment of chronic hepatitis B: AASLD 2018 hepatitis B guidance. Hepatology 2018;67:1560-1599.

9. Shin SK, Kim JH, Park H, et al. Improvement of liver function and non-invasive fibrosis markers in hepatitis B virusassociated cirrhosis: 2 years of entecavir treatment. J Gastroenterol Hepatol 2015;30:1775-1781.

10. Chen CF, Lee WC, Yang HI, et al. Changes in serum levels of HBV DNA and alanine aminotransferase determine risk for hepatocellular carcinoma. Gastroenterology 2011;141:12401248.

11. Kurokawa M, Hiramatsu N, Oze T, et al. Long-term effect of lamivudine treatment on the incidence of hepatocellular carcinoma in patients with hepatitis B virus infection. J Gastroenterol 2012;47:577-585.

12. Sarin SK, Kumar M, Lau GK, et al. Asian-Pacific clinical practice guidelines on the management of hepatitis B: a 2015 update. Hepatol Int 2016;10:1-98.

13. Chang TT, Lai CL, Kew Yoon S, et al. Entecavir treatment for up to 5 years in patients with hepatitis B e antigen-positive chronic hepatitis B. Hepatology 2010;51:422-430.

14. Hosaka T, Suzuki F, Kobayashi M, et al. Long-term entecavir treatment reduces hepatocellular carcinoma incidence in patients with hepatitis B virus infection. Hepatology 2013;58:98-107.

15. Suh B, Park S, Shin DW, et al. High liver fibrosis index FIB4 is highly predictive of hepatocellular carcinoma in chronic hepatitis B carriers. Hepatology 2015;61:1261-1268.

16. Paik N, Sinn DH, Lee JH, et al. Non-invasive tests for liver disease severity and the hepatocellular carcinoma risk in chronic hepatitis B patients with low-level viremia. Liver Int 2018;38:68-75.

17. Tseng TC, Liu CJ, Su TH, et al. Fibrosis-4 index helps identify HBV carriers with the lowest risk of hepatocellular carci- 
noma. Am J Gastroenterol 2017;112:1564-1574.

18. Nishikawa H, Nishijima N, Enomoto H, et al. Comparison of FIB-4 index and aspartate aminotransferase to platelet ratio index on carcinogenesis in chronic hepatitis $\mathrm{B}$ treated with entecavir. J Cancer 2017;8:152-161.

19. Tada T, Kumada T, Toyoda H, Tsuji K, Hiraoka A, Tanaka J. Impact of FIB-4 index on hepatocellular carcinoma incidence during nucleos $(\mathrm{t})$ ide analogue therapy in patients with chronic hepatitis B: an analysis using time-dependent receiver operating characteristic. J Gastroenterol Hepatol 2017;32:451-458.

20. Kim BK, Kim DY, Park JY, et al. Validation of FIB-4 and comparison with other simple noninvasive indices for predicting liver fibrosis and cirrhosis in hepatitis B virusinfected patients. Liver Int 2010;30:546-553.

21. Jin W, Lin Z, Xin Y, Jiang X, Dong Q, Xuan S. Diagnostic accuracy of the aspartate aminotransferase-to-platelet ratio index for the prediction of hepatitis B-related fibrosis: a leading meta-analysis. BMC Gastroenterol 2012;12:14.

22. Lu L, Yip B, Trinh H, et al. Tenofovir-based alternate therapies for chronic hepatitis B patients with partial virological response to entecavir. J Viral Hepat 2015;22:675-681.

23. Yip B, Chaung K, Wong CR, et al. Tenofovir monotherapy and tenofovir plus entecavir combination as rescue therapy for entecavir partial responders. Dig Dis Sci 2012;57:30113016.

24. Choi J, Kim HJ, Lee J, Cho S, Ko MJ, Lim YS. Risk of hepatocellular carcinoma in patients treated with entecavir vs tenofovir for chronic hepatitis B: a Korean nationwide cohort study. JAMA Oncol 2019;5:30-36.

25. Pang Q, Xu XS, Zhang JY, Qu K, Chen W, Liu C. FIB-4 as a prognostic model for patients with hepatitis B-associated hepatocellular carcinoma. Hepatology 2015;62:1325-1326. 